\title{
NEXT GENERATION NEUTRON SCINTILLATORS BASED ON SEMICONDUCTOR NANOSTRUCTURES
}

\author{
FINAL REPORT
}

$6 / 30 / 2008$

\subsection{SIGNIFICANCE, BACKGROUND INFORMATION, AND}

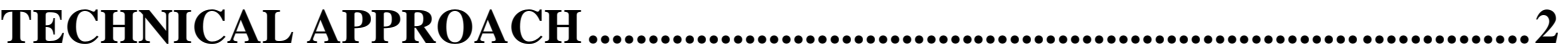

1.1 IDENTIFICATION AND SignifiCANCE OF THE PROBlEM OR OPPORTUNITY, AND

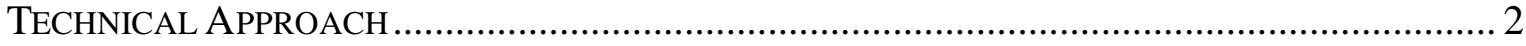

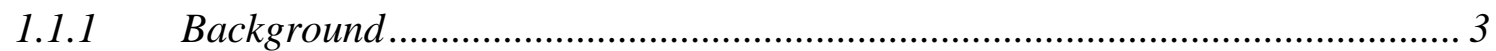

1.1.2 Technical Development of Nanostructured Scintillators ................................. 5

1.2 ANTICIPATED PUBLIC BENEFITS ................................................................... 6

1.3 DEgReE to Which Phase I has Demonstrated TeChNicAl FEASIBILITY ............ 6

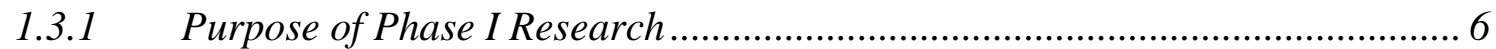

1.3.2 Results and Discussion .......................................................................... 7

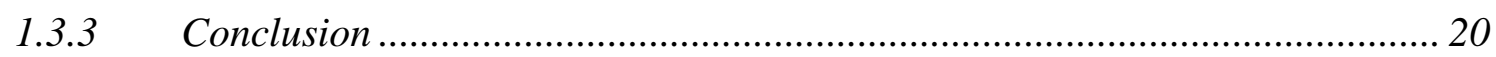

2.0 BIBLIOGRAPHY \& REFERENCES CITED ................................21 


\subsection{SIGNIFICANCE, BACKGROUND INFORMATION, AND TECHNICAL APPROACH}

\subsection{Identification and Significance of the Problem or Opportunity, and Technical Approach}

Neutron scattering techniques provide an excellent probe of structure and dynamics of condensed matter. During the past decade, appreciation of this technology has extended to such fields as nanotechnology, biology, polymer chemistry, nondestructive imaging and many others and is expanding rapidly. Today, neutron technology in the US lags behind that in Europe and Japan. Recent commission of a new high-power neutron source, Spallation Neutron Source (SNS) at Oak Ridge National Laboratory (ORNL), will allow the neutron scattering research to leap forward. The SNS will provide researchers with the most intense pulsed neutron source in the world. However, in order to capitalize on the improvement in neutron source intensity, parallel advances in neutron detection technology must be achieved.

A recent white paper provided a detailed description on the need for the improved neutron detector technology for high power pulsed neutron source such as SNS and JSNS (Cooper, 2003). The authors underscored the degrees of the problems by stating "Because of the long lead time for detector development, it is already too late to provide optimized detectors for the initial suite of SNS instruments. The detectors that will be installed initially will significantly restrict the performance of the instruments."

For many neutron scattering instruments, the detector specifications call for high rate capability, large area coverage, high spatial resolution, low gamma sensitivity, and costeffectiveness. There are currently no developed detector options that satisfy these demanding and frequently controversial requirements; however, one such detector holds much of that promise. It is the SNS neutron Anger Camera.

PartTec's service to SNS's Detector Group has been for the design and engineering of existing detector prototypes in ways that improve their manufacturability that allow concurrent engineering during manufacture and which improve the detectors' form, fit and function.

PartTec has developed, in Phase I of this project, a novel neutron scintillator consisting of a neutron absorber and $\mathrm{ZnS}$ semiconductor quantum dots (QD). The scintillator is faster than even the stringent requirement as stated in the white paper, i.e., the large area coverage combined with small pixel sizes, high neutron detection efficiencies, and 1- $\mu$ s time resolution eliminates all but the scintillator option (Cooper, 2003). PartTec's novel scintillator will have short light decay time ( $<30 \mathrm{~ns}$ ), high light yield, and high neutron to gamma discrimination ratio. Its short decay time will allow event-by-event sampling in time-of-flight (TOF) detection instruments for high flux pulse neutron sources with broadband wavelengths. 
Final Report Next Generation Neutron Scintillators based on Semiconductor Nanostructures

DOE Award: DE-FG02-07ER84884

\subsubsection{Background}

Scintillators with high light yield and short light decay time are crucial for improving neutron efficiency, spatial resolution, and detectability under high neutron flux. Table 1-1 includes a list of some commercially available scintillators that are frequently used in thermal neutron imaging detectors.

Table 1-1. Several commercial scintillators for thermal neutron detection (Crawford, 1992; Clergeau, 2002)

\begin{tabular}{|c|c|c|c|c|c|}
\hline Material & $\begin{array}{c}\text { Density of } \\
\text { Absorbed Nuclei } \\
\left(\mathbf{1 0}^{\mathbf{2 2}} / \mathbf{c m}^{\mathbf{3}}\right)\end{array}$ & $\begin{array}{c}\text { Scintillator } \\
\text { Efficiency (\%) }\end{array}$ & $\begin{array}{c}\text { Photon } \\
\text { Wavelength }\end{array}$ & $\begin{array}{c}\text { Photons per } \\
\text { Neutron (nm) }\end{array}$ & $\begin{array}{c}\text { Light Decay Time } \\
(\boldsymbol{\mu s})\end{array}$ \\
\hline${ }^{6} \mathrm{Li}$ glass(Ce) & 1.75 & 0.45 & 395 & $\sim 7,000$ & $\sim 0.1^{\mathrm{c}}$ \\
\hline${ }^{6} \mathrm{Lil}(\mathrm{Eu})$ & 1.83 & 2.8 & 470 & $\sim 51,000$ & $1.4^{\mathrm{d}}$ \\
\hline $\mathrm{ZnS}(\mathrm{Ag}) /^{6} \mathrm{LiF}^{b}$ & 1.18 & 10.8 & 450 & $\sim 175,000$ & $0.2,>10$ \\
\hline
\end{tabular}

${ }^{\mathrm{a}}$ Fraction of nuclear energy converted to useful photons

${ }^{\mathrm{b}}$ Applied Scintillation Technologies (AST), in a 4/1 ratio mixture

${ }^{c}$ Decayed to $10 \%$. It is also cited as 75 ns (van Eijk, 2004).

${ }^{\mathrm{d}}$ Apace Science (Commercial name)

Among commercial scintillators, the ${ }^{6} \mathrm{Li}$-glass is most transparent but has the lowest light yield. $\mathrm{ZnS}$ is a micrometer-sized powder and is mixed with ${ }^{6} \mathrm{LiF}$ and organic binder to form a scintillator layer. This layer is opaque to its own scintillation light and the high refractive index of $\mathrm{ZnS}: \mathrm{Ag}$ particles, and thus its thickness is limited to $<0.5 \mathrm{~mm}$, resulting in thermal neutron efficiency lower than $30 \%$. ${ }^{6} \mathrm{LiI}$ is hygroscopic and has slower response than ${ }^{6} \mathrm{Li}$ glass. The light yield per $\mathrm{MeV}$, which is different for capture reaction products of a thermal neutron and a gamma-ray, determines the discrimination ability between a neutron and gamma-ray with pulse-height analysis (van Eijk, 2004). In ${ }^{6} \mathrm{Li}$-glass, a $1.5 \mathrm{MeV}$ gamma ray produces the same number of photons as $4.8 \mathrm{MeV}$ heavy particles from a neutron capture event. Another aspect is the response time: in microparticle LiF/ZnS:Ag scintillators, the gamma ray response time is much shorter than the neutron response time, which offers the possibility of neutron/ $\gamma$-ray discrimination using pulse-shape analysis (Clergeau, 2002; van Eijk, 2004).

Neutron $/ \gamma$-ray discrimination characteristics of $\mathrm{ZnS}$-based detector were measured by Gorin, et al (2002). It was shown that neutron/ $\gamma$-ray ratio could be improved by introducing a lower cut of pulse height distribution, though at the cost of decreasing efficiency. A broad distribution of neutron pulse is observed from microparticle $\mathrm{ZnS} /{ }^{6} \mathrm{LiF}: \mathrm{Ag}$ scintillators (Gorin, 2002; Katagiri, 2004b), Neutron/ $\gamma$-ray characteristics of several novel neutron scintillators, including $\mathrm{Li}_{2} \mathrm{~B}_{4} \mathrm{O}_{7}$ glass and ${ }^{6} \mathrm{Li}$-loaded plastic scintillators were measured (Katagiri, 2004b). Though some of them demonstrated abilities for $\mathrm{n} / \gamma$ discrimination, the characteristics were significantly worse than that of microparticle $\mathrm{ZnS}$ scintillator.

The neutron Anger camera is composed of a ${ }^{6} \mathrm{Li}$-glass scintillator, a light coupler/guide, and an array of multi-anode PMTs with multiple readout channels. Light emitted from neutron scintillator is collected by PMT photocathodes and the neutron hit position is calculated based on the photon counts at pixels.

The typical cross-fiber scintillation neutron detector used in neutron scattering facilities works as follows. Neutrons are absorbed causing emitted scintillation photons from the 
scintillator, typically a $\mathrm{ZnS}: \mathrm{Ag} /{ }^{6} \mathrm{LiF}$ microparticle based composite. The photons travel to the intersection of two wavelength shifting (WLS) plastic optical fibers (POF) where longerwavelength light is produced and transported to photomultiplier tubes (PMTs) attached to the fibers. The neutron position is determined by the coincidence of signals from the PMTs (Crow, 2004).

Table 1-2 lists the detector requirements for several SNS instruments, demonstrating a crossfiber scintillator detector to be the best choice for those instruments. Table 1-3 lists the gap in performance specifications that must be bridged by future development work, especially the light yield, light decay time, and neutron capture efficiency.

Table 1-2. Detector requirements for selected SNS instruments (Cooper, 2004). Scintillator detector is the leading candidate for each of the listed instruments.

\begin{tabular}{|c|c|c|c|c|c|c|c|}
\hline Instrument & $\begin{array}{c}\text { Number of } \\
\text { Pixels }\end{array}$ & $\begin{array}{c}\text { Pixel area } \\
\mathbf{( \mathbf { c m } 2 )}\end{array}$ & $\begin{array}{c}\text { Max. } \\
\text { neutron } \\
\text { energy } \\
(\mathbf{e V})\end{array}$ & $\begin{array}{c}\text { Neutron } \\
\text { capture } \\
\text { efficiency } \\
(\mathbf{\%})\end{array}$ & $\begin{array}{c}\text { Gamma } \\
\text { efficiency }\end{array}$ & $\begin{array}{c}\text { Time } \\
\text { resolution } \\
(\boldsymbol{\mu})\end{array}$ & $\begin{array}{c}\text { Detector } \\
\text { count rate } \\
(\mathbf{1} / \mathbf{s})\end{array}$ \\
\hline $\begin{array}{c}\text { Powder Diffractometer } \\
\text { (POWGEN3) }\end{array}$ & 40,000 & 2.4 & 0.33 & 50 & $10^{-6}$ & 1 & $3.5 \times 10^{6}$ \\
\hline $\begin{array}{c}\text { Engineering } \\
\text { Diffractometer } \\
\text { (VULCAN) }\end{array}$ & 80,000 & 1.25 & 0.15 & 50 & $10^{-6}$ & 1 & $2.4 \times 10^{6}$ \\
\hline $\begin{array}{c}\text { Single-Crystal } \\
\text { Diffractometer (TOPAZ) }\end{array}$ & $5 \times 10^{6}$ & 0.01 & 0.35 & 50 & $10^{-6}$ & 10 & $3 \times 10^{5}$ \\
\hline Disordered Materials & 150,000 & 0.25 & 35 & 20 & $10^{-6}$ & 1 & $4.2 \times 10^{7}$ \\
\hline
\end{tabular}

The performance of Anger camera and wavelength shifting (WLS)-fiber detectors obtained up to now, as far as light yield per neutron and dynamic range are concerned, is marginal for many practical applications in SNS. Scintillators with higher light yield and shorter decay times, more efficient light transmission from scintillator to other components (light coupler, fiber, and PMT), and fast, multichannel readout systems are needed. 
Table 1-3. Comparison of desired vs. currently attainable detector parameters for SNS selected instruments (Cooper, 2004).

\begin{tabular}{|c|c|c|c|}
\hline Instrument & Parameter & Desired & Current \\
\hline \multirow{3}{*}{$\begin{array}{l}\text { Powder Diffractometer } \\
\text { (POWGEN3) }\end{array}$} & Neutron efficiency at $0.35 \mathrm{eV}(\%)$ & 50 & 30 \\
\hline & Detector cost $\left(\mathrm{K} \$ / \mathrm{m}^{2}\right)$ & 150 & 250 \\
\hline & $\begin{array}{l}\text { Transmission detector maximum } \\
\text { time average data rate }(1 / \mathrm{s})\end{array}$ & $3.4 \times 10^{7}$ & $1 \times 10^{6}$ \\
\hline \multirow{2}{*}{$\begin{array}{l}\text { Engineering Diffractometer } \\
\text { (VULCAN) }\end{array}$} & Spatial resolution $(\mathrm{mm})$ & 0.1 & 1 \\
\hline & $\begin{array}{c}\text { Transmission detector maximum } \\
\text { time average data rate }(1 / \mathrm{s})\end{array}$ & $5 \times 10^{7}$ & $1 \times 10^{6}$ \\
\hline \multirow{3}{*}{$\begin{array}{l}\text { Single-Crystal Diffractometer } \\
\text { (TOPAZ) }\end{array}$} & Spatial resolution $(\mathrm{mm})$ & $\leq 1$ & 3 \\
\hline & $\begin{array}{c}\text { Scintillator brightness } \\
\text { (photon/neutron) }\end{array}$ & 30,000 & 10,000 \\
\hline & $\begin{array}{c}\text { Dynamic range } \\
\text { (peak/background counts) }\end{array}$ & 1000 & 100 \\
\hline $\begin{array}{l}\text { Disordered Materials } \\
\text { (NOMAD) }\end{array}$ & $\begin{array}{c}\text { Detection efficiency for } 35 \mathrm{eV} \\
\text { neutrons }(\%)\end{array}$ & 20 & 5 \\
\hline
\end{tabular}

\subsubsection{Technical Development of Nanostructured Scintillators}

Nanostructured scintillators used for neutron detection can completely change the poor performance of standard scintillators by providing faster response time (1-100 ns), brighter scintillation, higher transparency, larger detection area, and lower fabrication cost. Recent attempts have been made for new neutron scintillators. ${ }^{6} \mathrm{Li}$ loaded silica-polymer nanocomposite gels were developed (Im, 2004; Kesanli, 2006), but no photon yield was reported. Letant (2006) used CdSe/ZnS core-shell QDs for detecting $\alpha$-particles (a daughter particle from the reaction of ${ }^{6} \mathrm{Li}$ and neutrons); however, only a very weak pulse was seen in the pulse height spectrum, due to very low QDs fraction in porous glass matrix. QD/organic semiconductor scintillator for radiation detection was proposed by Campbell and Crone (2006), and Eu-activated nanostructured ceramic scintillators were used as Xray storage phosphors by Johnson, et al. (2006), but none of these materials have been demonstrated for thermal neutron detection. Though the sol-gel method was routinely used for synthesizing $\gamma$ scintillators as summarized by Mansuy et al (2004), no successful sol-gel neutron scintillator was reported. New strategies must be adopted to improve the performance of QD based neutron scintillators. That is the objective of the Phase II project.

PartTec has chosen $\mathrm{ZnS}$ QDs as the scintillation component based on the following reasons:

1. They have short light decay times $(\sim 10 \mathrm{~ns})$ under UV excitation, which allows them to have single neutron counting ability even under high neutron flux.

2. They have peak emission wavelengths $(420-440 \mathrm{~nm})$ match the absorption band of standard WLS fibers or photomultipliers (PMT), as shown in Figure 1-2 and Figure 1-3. 
3. The excitons in $\mathrm{ZnS}$ have a small Bohr radius $(2.5 \mathrm{~nm})$ and a relatively large binding energy (36 meV), allowing them to be confined in QDs with high stability, and therefore high luminescence quantum yield.

4. The material characteristics of $\mathrm{ZnS}$ result in intraband level spacing of 100-300 meV in ZnS QDs, one order of magnitude higher than LO phonon energy ( 20-40 meV). That leads to low charge cooling rate and non-radiative recombination rate, and facilitates the radiative recombination.

In summary, brighter, more transparent and faster scintillators need to be developed. $\mathrm{ZnS}$ quantum dots, which provide short decay times $(\sim 10 \mathrm{~ns})$ and high quantum yields as demonstrated in the Phase I results of this project, are an excellent replacement to ${ }^{6} \mathrm{Li}$-glass, or microparticle ZnS-based neutron scintillators.

\subsection{Anticipated Public Benefits}

PartTec's scintillator has great potential to replace commercial neutron scintillators that are utilized in Anger cameras used for the Single Crystal Diffractometer (SCD, also called TOPAZ) and the Macromolecular Neutron Diffractometer (MaNDi) that are being commissioned at SNS. It may also impact other instruments including Disordered Materials Diffractometer (NOMAD), and Spallation Neutrons and Pressure Diffractometer (SNAP), High-Resolution General Purpose Powder Diffractometer (POWGEN3) and the Engineering Diffractometer (VULCAN).

The proposed QD neutron scintillators and associated electronics will also be widely applied in neutron imaging and dosimetry, homeland security, industrial testing, nuclear and highenergy physics, and medical physics. Moreover, the QD processes developed in this project will also benefit future development of nanostructured optoelectronic devices such as lightemitting devices (LEDs), lasers, memories, spintronic devices, photodetectors, and solar cells, to name a few.

\subsection{Degree to which Phase I has Demonstrated Technical Feasibility}

\subsubsection{Purpose of Phase I Research}

The original goal of this SBIR project was to develop highly bright, gamma-free nanostructured thermal neutron scintillators consisting of semiconductor quantum-dots (QD), a neutron absorber $\left({ }^{6} \mathrm{LiF}\right)$, and a polymer matrix. In Phase I, PartTec planed to demonstrate that ZnS QDs have high luminescent efficiencies using capping and dopant engineering, and $\mathrm{ZnS}$ QDs/polymer composites have better optical transparency than conventional $\mathrm{Ag}: \mathrm{ZnS} /{ }^{6} \mathrm{LiF}$ microparticle neutron scintillators. PartTec planned, in particular, to measure the photoluminescence (PL) spectra, PL quantum yield (QY), optical absorption spectra of both $\mathrm{ZnS}$ quantum dots in solution and in composites. The new scintillator would overcome the low light yield and long light-pulse decay time of conventional scintillators. We emphasized that light yield, transparency, and light decay time are the key factors for new neutron scintillators. Below is the Phase I proposal synopsis:

1. Enhance luminescent efficiency by improving charge confinement efficiency of QDs.

a) Fabricate $\mathrm{ZnS}$ QDs in solutions, and optimize fabrication processes. 
Final Report Next Generation Neutron Scintillators ～DOE Award: DE-FG02-07ER84884 based on Semiconductor Nanostructures

b) Measure PL quantum yield, PL lifetime, and optical absorptions of QD in solution.

c) Characterize QDs using microscopic and other spectroscopic methods.

d) Decide to use undoped or doped QDs with suitable dopants $\left(\mathrm{Ag}^{+}, \mathrm{Ce}^{3+}\right.$, $\left.\mathrm{Eu}^{3+}\right)$.

e) Choose capping (or surfactants) among organic (octalymine, oleic acid, polymer) and inorganic (such as $\mathrm{SiO}_{2}$ ) agents.

f) Test stability of QDs under ambient UV light radiation or annealing.

2. Increase the transparency of QD based scintillators.

a) Increase Stokes-shift of QDs.

b) Fabricate QD/polymer composite films.

c) Measure optical absorption of QDs and composite films.

d) Increase light extraction efficiency of composite films.

3. Measure the pulse height or light yield of QD scintillator under neutron and/or gamma-ray radiation.

4. Research scintillation mechanism.

a) Find the root-cause and correlation of photoluminescence and radioluminescence.

b) Choose the light emitting candidate between defects and dopants.

During the Phase I period, PartTec performed experiments on fabrication and characterization of ZnS QDs. Some work was beyond the original tasks, but the original goal was kept. The core questions emphasized during the course of the Phase I project include the following:

1. What is the size of fabricated ZnS QDs?

2. How bright and how fast is the luminescence of $\mathrm{ZnS}$ QDs?

3. How stable is $\mathrm{ZnS}$ QDs?

4. Which dopant or capping agent is most suitable?

5. Can we reduce the light absorption of composite films at luminescent wavelengths?

\subsubsection{Results and Discussion}

\subsubsection{QD Synthesis}

Undoped and $\mathrm{Ag}^{+}, \mathrm{Ce}^{3+}, \mathrm{Eu}^{3+}$ doped nanocrystals were prepared using two methods. The first method was rapid, microwave-assisted, polyol-mediated synthesis (Feldman, 2003). To prepare this material, $0.0227 \mathrm{~g}$ zinc acetate, $0.011 \mathrm{~g}$ thiourea (Sigma-Aldrich) and a preset amount of $\mathrm{AgCl}_{3}, \mathrm{CeCl}_{3}$, or $\mathrm{EuCl}_{3}$, were dissolved in $20 \mathrm{ml}$ ethylene glycol (EG) and $4 \mathrm{ml}$ water. The mixture, after added coupling agent (surfactant) poly(vinyl pyrrolidone) (PVP) or ethylenediamine (EDA), was then rapidly heated using microwave radiation (300W) to 150 ${ }^{\circ} \mathrm{C}$ for 2-3.5 min. The particle size, typically above $10 \mathrm{~nm}$, was adjusted by heating time and temperature. The surface of growing particles was immediately complexed by coupling agent and EG (as a chelating agent) and agglomeration was prohibited.

The second method used co-precipitation, which directly produces ZnS QDs (or nanocrystals called by chemists) with smaller diameters $(4-10 \mathrm{~nm})$ at room temperature. Inorganic 
precursors of $\mathrm{Zn}^{2+}$ and $\mathrm{S}^{2-}$, dopant ions and capping molecules, were dissolved in aqueous medium. Undoped and $\mathrm{Eu}^{3+}$ (or other metal ions) doped nanocrystals were prepared. A water solution of zinc acetates, suitable $\mathrm{EuCl}_{3}$ and surfactant [EDA, PVP, or sodium polyphosphate (PP)] were pre-mixed under stirring. A water solution of $\mathrm{Na}_{2} \mathrm{~S}$ was added into the above mixture under stirring, leading to the formation of $\mathrm{ZnS}$ QDs capped with surfactants. For $\mathrm{ZnS}$ shell growth, the above process was repeated again without adding $\mathrm{EuCl}_{3}$ solution, and only $1 / 5$ volume of precursor and surfactant solutions was used. For $\mathrm{SiO}_{2}$ shell growth, $\mathrm{ZnS}$ QDs were centrifuged from the original solution; tetraethyl orthosilicate (TEOS) in ethanol solution was added into $\mathrm{ZnS}$ water solution.

For optical measurements, QDs were usually centrifuged from original solution to remove the residue, and were diluted with water or ethanol. Sometimes for a quick turn-around of optical or microscopic measurements, the QD samples in the original synthesis solution were used.

Microscopic and optical measurements were performed in microscopy facility of Indiana Molecular Biology Institute (IMBI), and PBIF (Physical Biochemistry Instrumentation Facility), Indiana University, Bloomington. A JEOL 1010 transmission electron microscope (TEM) was used at $80 \mathrm{kV}$. Two typical TEM images of ZnS QDs are shown in Figure 1-1. The main products are spherical or quasi-spherical nanoparticles with the average size of about $10 \mathrm{~nm}$. Figure 1-1(b) shows a little agglomeration of smaller QDs due to evaporation of water. The more precise values of QD sizes should be obtained based on the band-gap values from optical spectroscopic methods.
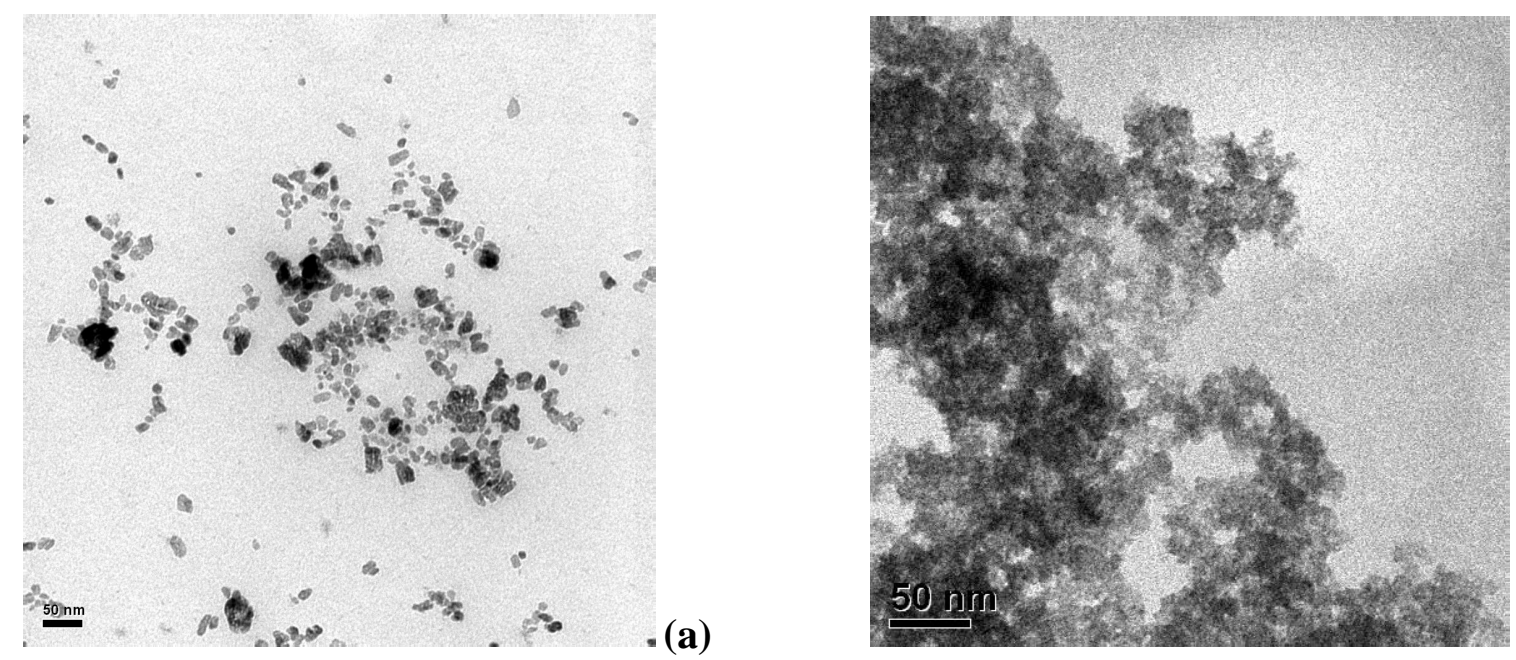

(b)

Figure 1-1. Example of TEM images for (a) an undoped ZnS QD sample. (b) Eu: ZnS (0.25\% Eu) core-shell QDs. The scale bar is $50 \mathrm{~nm}$.

\subsubsection{Optical measurements}

\section{Photoluminescence (PL) and $U V$ absorption}

UV absorption measurement was performed for dilute $\mathrm{ZnS}$ QD solution $\left(<10^{-3} \mathrm{M}\right)$ in water or ethanol with a Perkin-Elmer Lambda 19 spectrometer. PL and PL excitation (PLE) were performed on a Perkin-Elmer LB-50 fluorometer with a xenon lamp as light source. Both 
water and ethanol have negligible optical absorption and emission in the wavelength regions of interest.

Figure 1-2(a) shows UV absorption spectra of a series of Eu-doped ZnS QDs (Eu=0-0.50 at $\%$ ) in water solution. The small shoulders or bumps near $310-320 \mathrm{~nm}$ are due to excitoninduced absorption (or quantum confinement), are good indications of the band-gap $\left(E_{\mathrm{g}}\right)$ of QDs, since the exciton binding energy is very small $(\sim 40 \mathrm{meV})$ compared with $E_{\mathrm{g}}$. The exciton-induced absorption peak, an indication of exciton formation under UV excitation, can be seen more clearly at 310-320 nm in PLE spectra in Figure 1-2(b).

Besides the band-edge absorption peak at PLE spectra, there is a common peak at $235 \mathrm{~nm}$ $(5.28 \mathrm{eV})$, whose position is independent of the capping agents or doping species $(\mathrm{Ag}, \mathrm{Ce}$, $\mathrm{Eu})$. Previously the $235 \mathrm{~nm}$-peak was assigned to the electronic transitions in PVP surfactants (Manzoor, 2004). That is not the case, since the position of this peak is hardly changed by different capping layers, therefore it comes from $\mathrm{ZnS}$ QD itself. Other weaker peaks at 253, 260, $275 \mathrm{~nm}$ are also observed in PLE spectra, presumably due to other allowed electronic transitions between various energy levels (orbits) in valence band and conduction band in ZnS QDs (Cardona, 1965). However, they are not from capping agents because of their presence in all Eu:ZnS capped with PP, EDA, and PVP. Due to quantum confinement, we expect these peaks are shifted compared with those in $\mathrm{ZnS}$ bulk or microparticles. In addition, the band-gap values $\left(E_{g}=3.8-4.1 \mathrm{eV}\right)$ of the Phase I ZnS QD samples indicate the cubic (spherite) structure is formed. From the observed $E_{g}$, the average QD diameter $D$ of about $4.0 \mathrm{~nm}$ is estimated, based on $E_{\mathrm{g}}(\mathrm{D})$ relation in the effective mass approximation (Brus, 1984; Lippens, 1989). The band gaps of QDs are blue-shifted compared with that of cubic bulk $\mathrm{ZnS}$ crystals at $337 \mathrm{~nm}\left(E_{\mathrm{g}}=3.68 \mathrm{eV}\right)$.
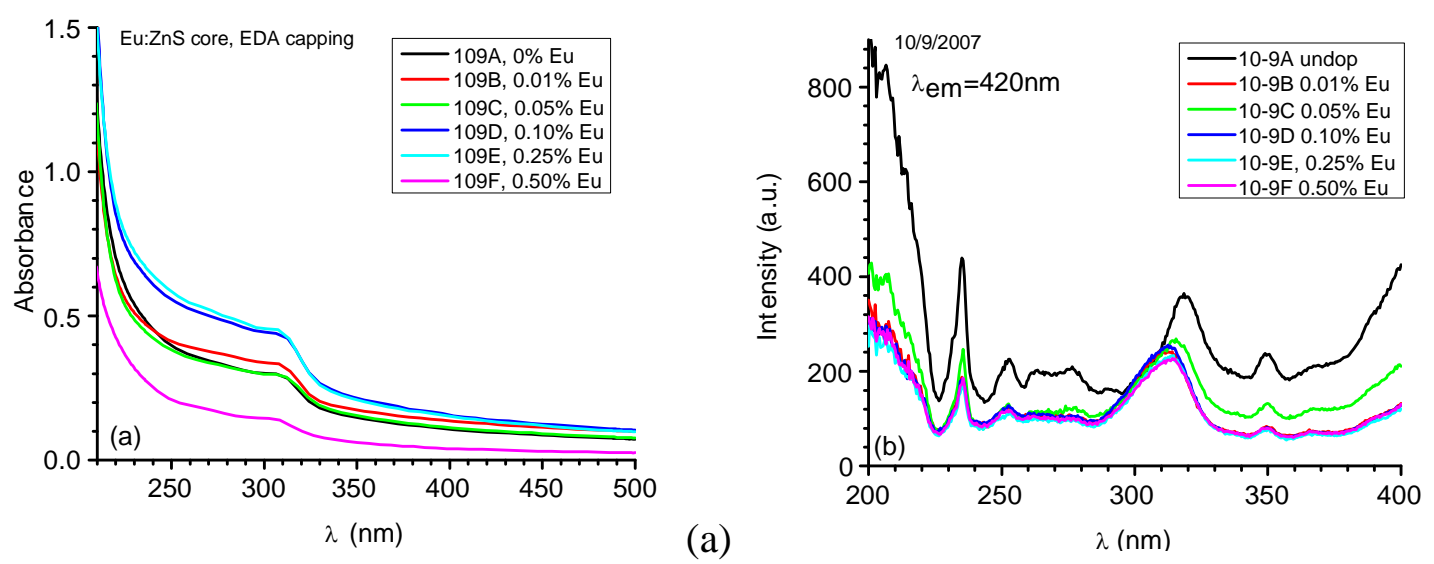
Final Report Next Generation Neutron Scintillators ～DOE Award: DE-FG02-07ER84884 based on Semiconductor Nanostructures
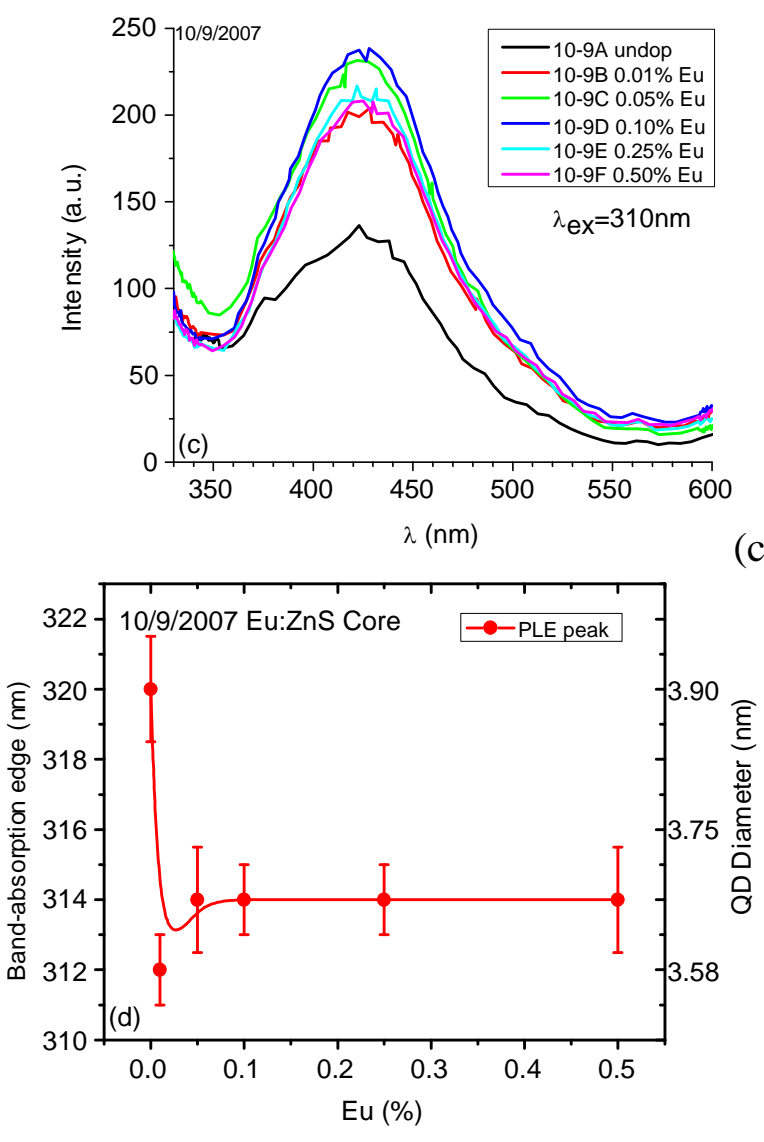

(d)

Figure 1-2. (a) absorbance, (b) PLE at emission wavelength of 420nm, (c) PL at excitation wavelength of $310 \mathrm{~nm}$, (d) band-absorption peak (or average diameter) versus Eu apparent doping concentration for Eu:ZnS QDs. The curve in (d) is used to guide the eye.

In Figure 1-2(b) and (d), the small change in exciton-induced absorption peak versus $\mathrm{Eu}^{3+}$ content may be explained by QD growth mechanism (Yin, 2005). In the mixed precursor solution a small amount of impurities or dopants changes the Gibbs energy of the system and the critical nucleus size. The final size of QDs is determined by the delicate balance between nucleation and dissolution. The change in critical nucleation size due to $\mathrm{Eu}^{3+}$ concentration leads the change in average quantum dot size.

PL peaks of ZnS QDs shown in Figure 1-2(c) are broad and can be decomposed into several narrower peaks. Low temperature PL measurement is expected to reduce the peak widths due to weaker vibronic (phonon) processes at low temperatures (Dean, 1982); however, such a measurement was not performed because of its irrelevance to scintillation at room temperatures. Nevertheless, as in the bulk $\mathrm{ZnS}$, the dominant defect involving the violet-blue emission in QDs is sulfur vacancies $\left(\mathrm{V}_{\mathrm{S}}\right)$, which are the most stable ones and create shallow donor levels below the conduction band (CB) dopant (Wright, 1995; Khosravi, 1995). Due to the size distribution of QDs and quantum confinements, the energy levels of defects $\mathrm{V}_{\mathrm{S}}$ also have a wider distribution and its deeper in $\mathrm{ZnS}$ QDs than in bulk ZnS. Possibly electron-hole pairs created by excitation are trapped at these defects, where they recombine and generate luminescence. 
While dopant ions such as $\mathrm{Ag}^{+}$may create a deep acceptor level in $\mathrm{ZnS}$ and trap excitons, $\mathrm{Ag}^{+}$emission may overlap with a defect emission in ZnS QDs as in the bulk (Era, 1968). As for the rare-earth (RE) dopants, RE ion intra-ion emission is induced in many host crystals when the ionized carriers are captured by RE. However, in our PL and PLE measurements PartTec did not observe the emission directly from rare-earth ions. Three reasons are likely responsible for this and they incude the following:

1. There is thermal dissociation of RE bound exciton (BE) at room temperature (Swiatek, 1990), which reduce the efficiency of energy transfer and thus quenching the RE intraion emission at room temperature.

2. There may be an efficient energy transfer from RE BE to impurities or trapping centers in NCs, competing with energy transfer from BE to RE core states (Swiatek, 1990).

3. RE ions may not be doped inside NCs but on the surface of NCs (Bol, 2002), allowing defect emission dominant.

It is possible to verify the first and second hypothesis in our samples by measuring temperature-dependence of PL intensity. Nevertheless, we observed very similar PL and PLE spectra of $\mathrm{Eu}^{3+}$ and $\mathrm{Ce}^{3+}$ doped $\mathrm{ZnS}$ QD samples, which supports the third hypothesis and that defect emission from these samples is the most important.

In Figure 1-2(a-c), the Stokes shifts of Eu:ZnS QDs, i.e., the gaps between absorption edges $(300-320 \mathrm{~nm})$ and $420 \mathrm{~nm}$ emission peaks, are $\sim 1 \mathrm{eV}$ and large enough for avoiding the selfabsorption of scintillation light by QD themselves. Our results clearly show the advantage of QD scintillator over commercially available ZnS microparticle scintillators in terms of Stokes shifts.

On the surface of QDs, there are a large number of defect states which act as non-radiative passageways for the ionized (or second, delta) electrons since the co-ordination number around the atoms is less than that inside bulk. Covering the QD surface with an inorganic shell or organic ligand molecules may bring about passivation of vacancies, stabilize colloidal suspension, and improve quantum confinement efficiency.

As expected, capping layers influence the peak positions and profiles of PL spectra. Figure 1-3 shows PL spectra of nine Eu:ZnS samples with apparent dopant concentration of 0$0.10 \%$. Obviously, ethylenediamine (EDA) capping produces stronger UV-violet emission than poly(sodium phosphate) (PP) and poly(vinyl pyrrolidone) (PVP), which are much longer chains than EDA. Compared with doped QDs with the same type of capping agents, the undoped $\mathrm{ZnS}$ QD samples normally have lower PL intensity or quantum efficiency as seen in Figure 1-3. We have normalized spectrum by absorbance at $310 \mathrm{~nm}$. 
Final Report Next Generation Neutron Scintillators ～DOE Award: DE-FG02-07ER84884 based on Semiconductor Nanostructures

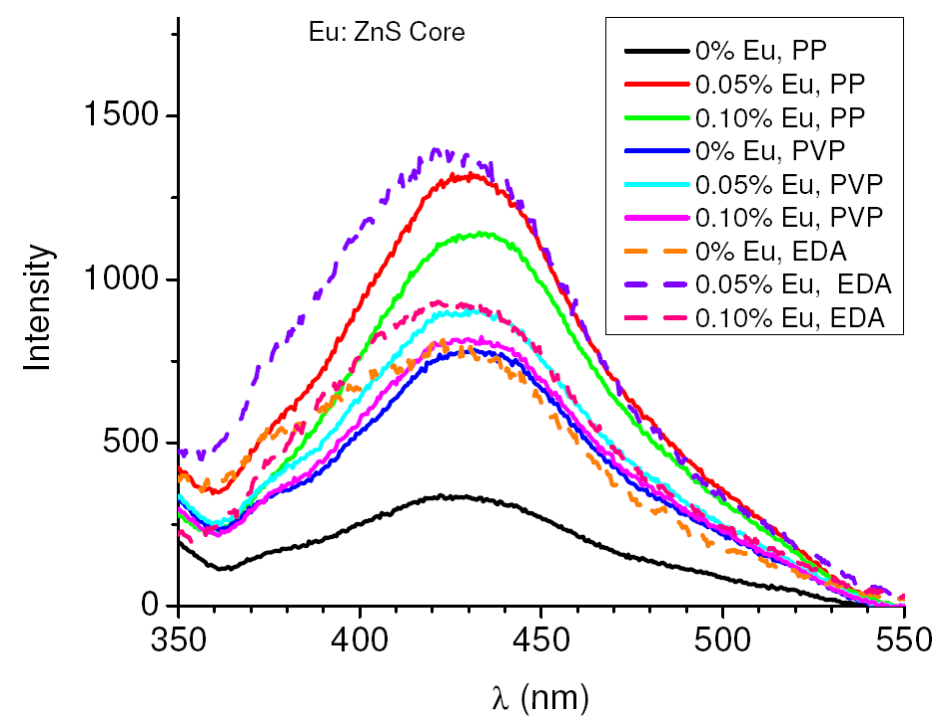

Figure 1-3. PL for Eu:ZnS capped with EVA, PVP, and PP. The excitation wavelength for PL is $310 \mathrm{~nm}$. The PL intensity was normalized by the absorbance at $310 \mathrm{~nm}$.

\section{PL lifetime measurement}

PL decay curves at $420 \mathrm{~nm}$ peak emission were measured using a $355-\mathrm{nm}$ laser, the $3^{\text {rd }}$ harmonic line of Nd:YAG laser, a photomultiplier tube and an oscilloscope. The laser pulse width is around $10 \mathrm{~ns}$. Figure 1-4 is an example of PL lifetime profile at $420 \mathrm{~nm}$ emission for a Eu:ZnS QD sample in water solution. The apparent decay lifetimes is $\sim 17 \mathrm{~ns}$, much smaller than the 1-10 $\mu$ s component of Ag:ZnS microparticles in conventional scintillators. Our lifetime result is also in the same order of magnitude of 2-60 ns for undoped and $\mathrm{Cu}$ doped ZnS QDs reported by Khosravi, et al (1995).

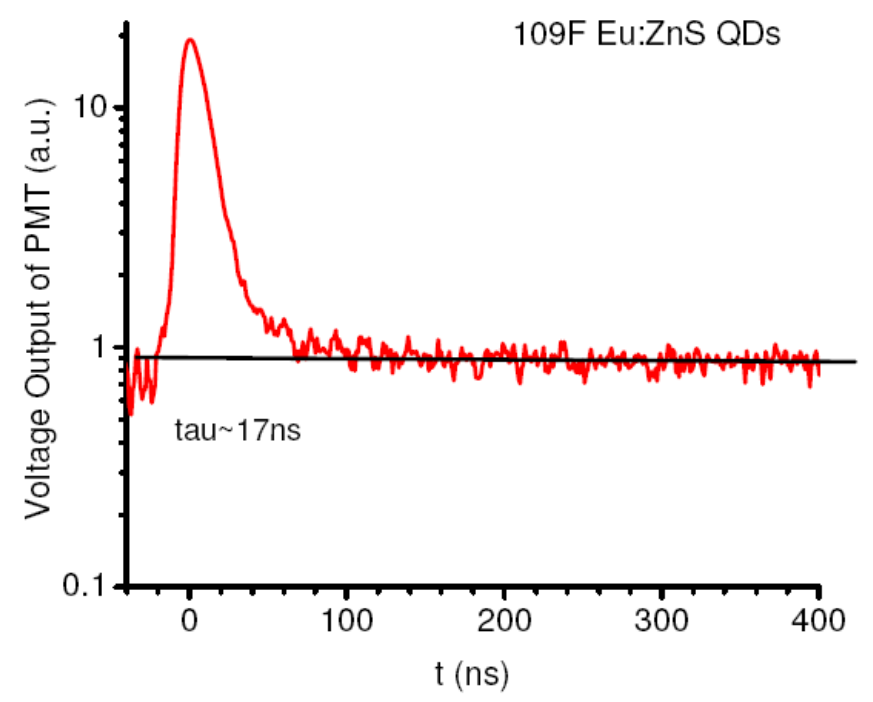

Figure 14. PL lifetime decay profile for an Eu:ZnS QD sample. 
Final Report Next Generation Neutron Scintillators based on Semiconductor Nanostructures

DOE Award: DE-FG02-07ER84884

\section{Photoluminescence quantum yields (QYs) of ZnS QDs}

PL quantum yield (QY), $\eta$, defined as the number of emitted photons created in QDs by absorption $100 \mathrm{UV}$ photons, was measured relative to Rhodamine 6G (with a concentration lower than $10^{-4} \mathrm{M}$ ) in ethanol (Cumberland, 2002; Demas, 1971), and was calculated by

$\eta=\eta^{\prime}\left(I / I^{\prime}\right)\left(A^{\prime} / A\right)\left(n / n^{\prime}\right)^{2}$

Where $I$ (sample) and $I^{\prime}$ (standard) are the integrated emission peal areas, $A$ (sample) and $A^{\prime}$ (standard) are the absorbances at the excitation wavelength, $n$ (sample) and $n$ ' (standard) are the refractive indices of solvents, and $\eta^{\prime}$ (standard) $=95 \%$ (Kubin, 1982).

Capping layers strongly influence the QY. Figure 1-5 shows the QY values for a series of $\mathrm{Eu}$ : ZnS QDs with surfactant EDA, PVP, or sodium polyphosphate (PP). Obviously PVP can provide the most efficient surface passiviation (Manzoor, 2004). Though PP capping for ZnS QDs is better than poly(vinylbutyral) (PVB), poly(vinyl alcohol) (PVA) (Bol, 2001), PP looks worse than PVP for core-shell samples, as shown in Figure 1-5. In Section 2.4, we see that inorganic $\mathrm{SiO}_{2}$ provides even better capping than solely EDA, PP, or PVP surfactant.

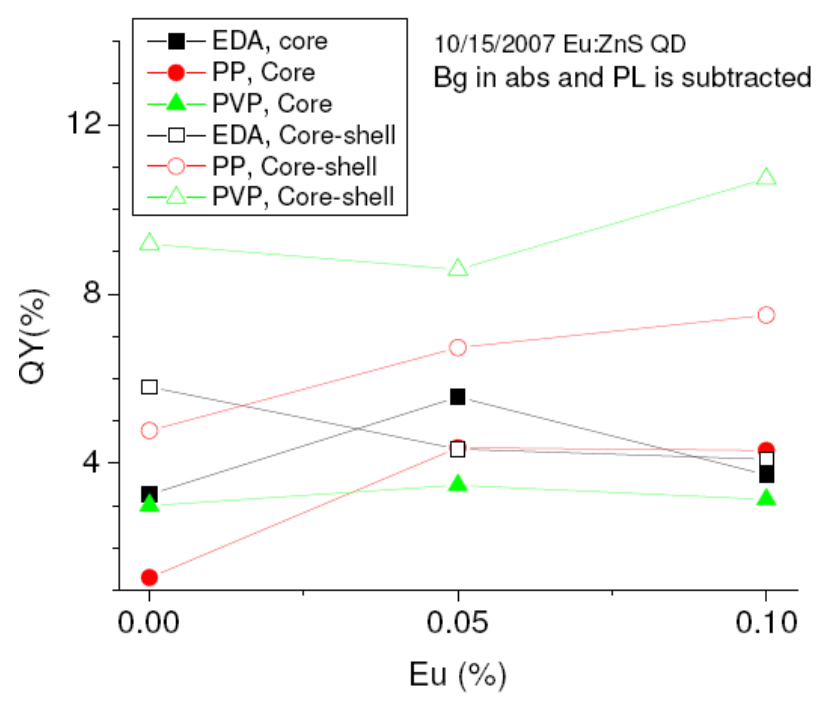

Figure 1-5. QYs for Eu:ZnS QDs with surfactants EDA, PVP, or PP. The PL spectra for the Eu:ZnS QD core samples are shown in Fig.4. "Core" means Eu:ZnS/surfactant QDs, while “Core-shell” means Eu:ZnS core/surfactant/ZnS shell QDs.

Compared with core QDs without shell (capping agents), capped QDs have larger luminescence quantum yield, due to the surface passivation of QDs. The high quantum yield $(\sim 10 \%)$ among these series of samples is close to the previously reported highest value of 10.2\% for Ag:ZnS QDs (Jian, 2006), but much higher than 4.5\% for Mn:ZnS QDs (Bol, 2001).

The fabrication condition, including capping, temperature, initial concentration of precursors (Yin, 2005), and even the mixing speed of precusors may strongly influence the PL QY.

Figure 1-6 shows the Eu-concentration dependence of another batch of core and core-shell samples, where the shell is a $\mathrm{ZnS}$ layer and the obtained samples are called isocrystalline QDs. The highest QY of Eu:ZnS QDs, 12\%, is higher than that in Figure 1-5, indicating the 
Final Report Next Generation Neutron Scintillators ～DOE Award: DE-FG02-07ER84884 based on Semiconductor Nanostructures

improvement in fabrication process. However, the $\mathrm{ZnS}$ shell does not improve the QY as compared with the pure core samples, indicating the very limited effect of $\mathrm{ZnS}$-shells after the QY is improved at a certain level (6-10\%) as seen in both Figure 1-5 and Figure 1-6.

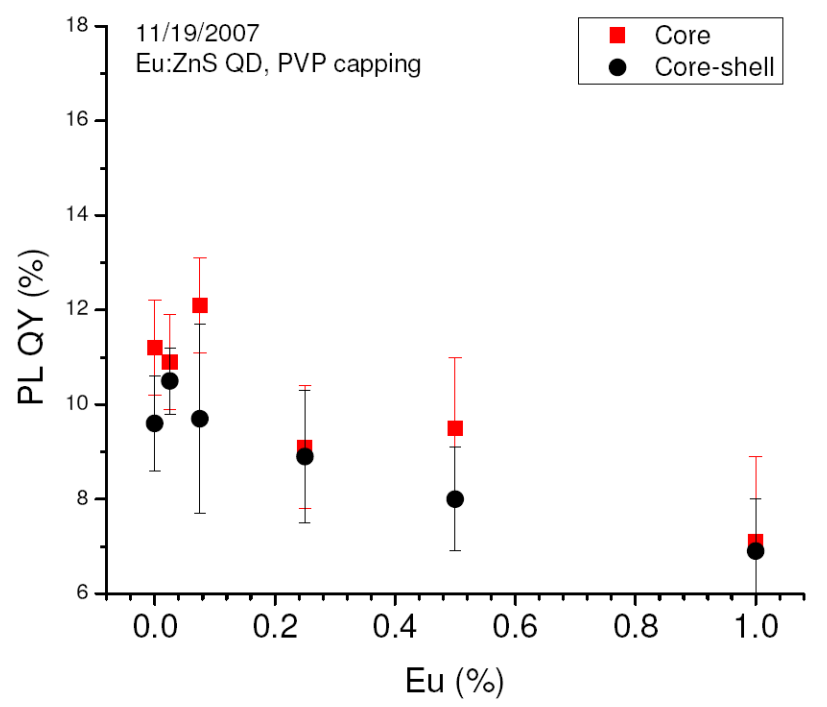

Figure 1-6. QYs for Eu:ZnS QDs capped with PVP as a function of Eu concentration. The core samples are Eu:ZnS/PVP QDs while the core-shell samples are Eu:ZnS/PVP/ZnS QDs. Error bar is based on QYs at several excitation wavelengths near $300 \mathrm{~nm}$.

Fig.7 is the comparison of quantum yields of several doped ZnS QD samples prepared under the same condition except that dopants were changed and one Eu:ZnS/PVP QD sample is overcoated with $\mathrm{SiO}_{2}$ shell. Obviously, the $\mathrm{Eu}^{3+}$-doped sample has the highest quantum efficiency than $\mathrm{Ag}^{+}$or $\mathrm{Ce}^{3+}$ doping. $\mathrm{SiO}_{2}$ shell can also provide good protection to $\mathrm{ZnS}$ QDs.

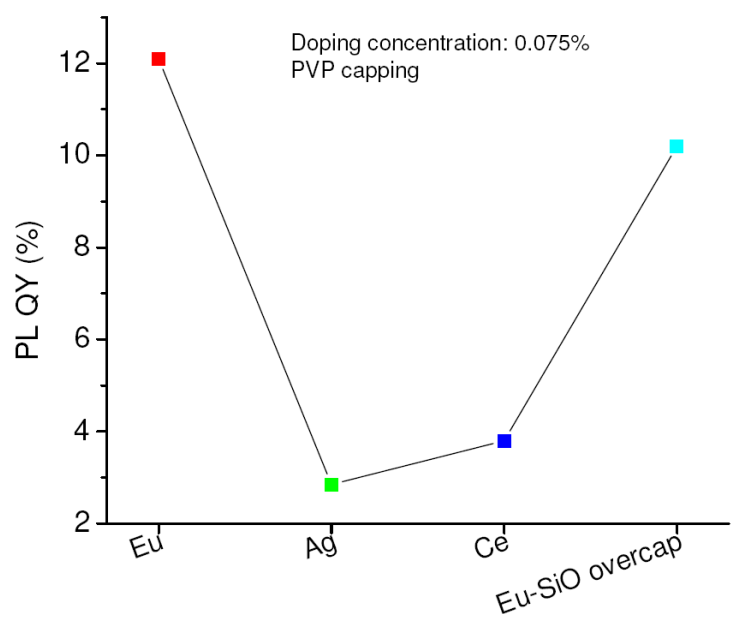

Figure 1-7. Comparison of quantum yields for $\mathrm{ZnS}$ QDs doped with $0.075 \% \mathrm{Eu}, \mathrm{Ag}$ and $\mathrm{Ce}$. All QDs are capped with PVP. The result for a Eu-doped ZnS QD sample overcapped with $\mathrm{SiO} 2$ shell is also included.

\section{Stability of ZnS QDs}


PartTec monitored the stability of ZnS QDs in solution by measuring UV absorption, PL, and quantum yields. A typical change in UV absorption spectrum for an undoped ZnS QDs capped with PVP is shown in Figure 1-8(a). The exciton absorption peak is slightly shifted to a longer wavelength and the absorbance is decreased. This is due to the dynamic balance between dissolution and nucleation in solution, as mentioned previously. Many samples have the similar tendency as in Figure 1-8(a).

Interestingly, we observed the change of UV absorption, PL, PLE, and PL quantum yield for a $\mathrm{SiO}_{2}$ capped $\mathrm{ZnS}$ QD sample after aging in solution for 46 days [Figure 1-8(b)]. Although the size of QD is hardly changed as seen from the exciton absorption edge (300nm) in PLE spectrum, the PL intensity is increased and peak is narrower, indicating the distribution of defect energy levels is narrower with the elapse of time. Quantum yield of this $\mathrm{SiO}_{2}$ capped sample is increased from $9.2 \%$ to $17.0 \%$ after 46 days aging. Meanwhile, the quantum yield of another Eu-doped $\mathrm{ZnS} / \mathrm{SiO}_{2}$ sample was unchanged. Nevertheless, this result indicates that $\mathrm{SiO}_{2}$ shell provides the excellent structure stability to the surface of $\mathrm{ZnS}$ QDs.
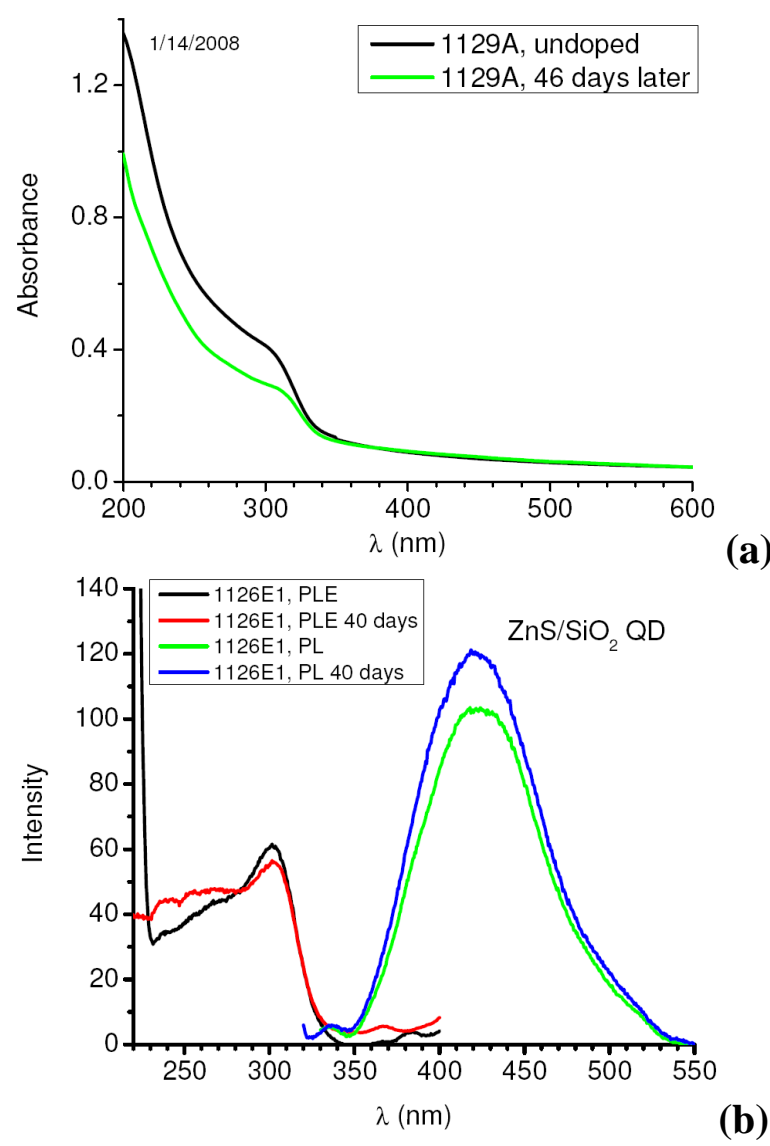

Figure 1-8. Change of absorption spectrum of an undoped ZnS QD sample in solution before and after 46 days aging. b). Changes in PLE and PL spectra for a $\mathrm{ZnS} / \mathrm{SiO} 2 \mathrm{QD}$ sample after 40 days in water-ethanol solution. PL and PLE is measured at $\lambda \mathrm{ex}=300 \mathrm{~nm}$ and $\lambda \mathrm{em}=420 \mathrm{~nm}$, respectively. Two PL spectra were normalized by the absorbance at $300 \mathrm{~nm}$ based on Eq. (1).

A more systematic measurements on the aging effects of ZnS QDs in water-ethanol solution with the same type of PVP capping but different dopants were performed. Figure 1-9 shows 
Final Report Next Generation Neutron Scintillators ～DOE Award: DE-FG02-07ER84884 based on Semiconductor Nanostructures

the change of quantum yield after 40-60 days aging in ambient conditions at room temperature. Though Ag-, Ce-doped and undoped ZnS QDs are stable within experimental errors, Eu-doped QDs show a large decrease in quantum yield. Nevertheless, Eu:ZnS QDs with $\mathrm{SiO}_{2}$ shell are very stable in deed. Furthermore, the sample of undoped $\mathrm{ZnS}$ QDs with $\mathrm{SiO}_{2}$ shell has the highest quantum yield $(17 \%)$ we obtained so far. This result indicates that aging (or annealing at certain temperature) may be effective in improving quantum yields.

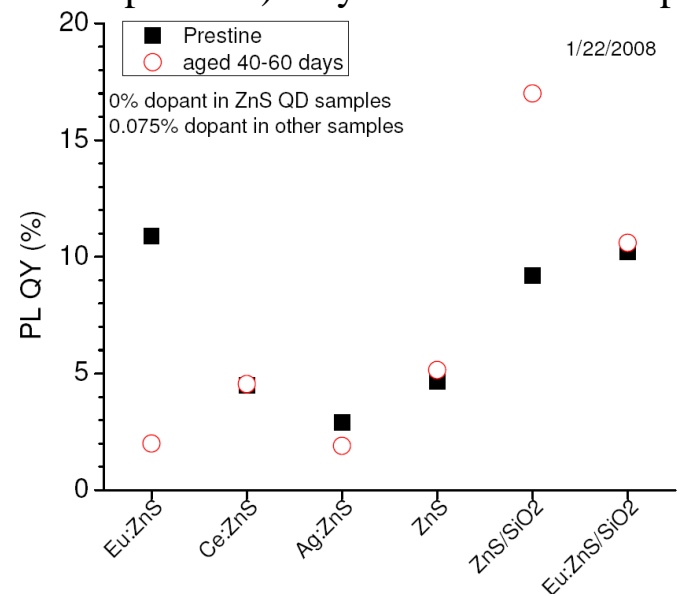

Figure 1-9. Variations of PL quantum yield in room-temperature aging for several QD samples. All QDs are capped with PVP. Two SiO2 overcapped samples were fabricated after PVP capping

\section{UV absorption properties of ZnS QD/polymer nanocomposite films}

$\mathrm{ZnS} / \mathrm{LiF}$ nanocomposite films were cast on glass from the mixture of $\mathrm{ZnS}$ QDs, LiF nano(NP) or micro-particles (MP), and poly(vinyl alcohol) (PVA) matrix in water-ethanol solution. For initial optical absorption measurement only, ${ }^{7} \mathrm{LiF}$ MPs (diameter $5 \mu \mathrm{m}$ ) or NPs (diameter $\sim 50-100 \mathrm{~nm}$ ) were used. Figure 1-10 and Figure 1-11 show the optical absorption coefficient versus wavelength for sample films. The concentration by weight of $\mathrm{LiF}, \mathrm{ZnS}$ and PVA matrix before casting are listed in the figures. 
Final Report Next Generation Neutron Scintillators ～DOE Award: DE-FG02-07ER84884 based on Semiconductor Nanostructures

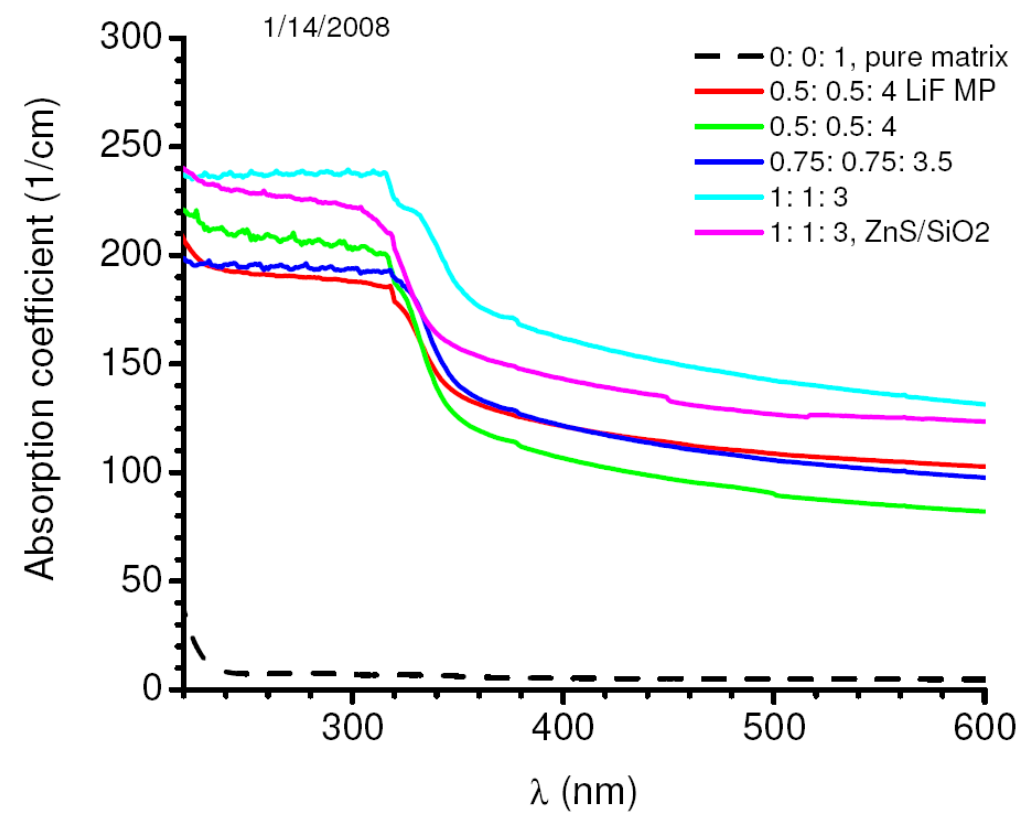

Figure 1-10. Optical absorption coefficient of several nanocomposite films with thickness of 0.1-0.3 mm. The weight ratio of 7LiF: $\mathrm{ZnS}$ : polymer is indicated in the legend. MP represents microparticle, and $\mathrm{ZnS} / \mathrm{SiO} 2$ means $\mathrm{ZnS} / \mathrm{PVP} / \mathrm{SiO} 2$ structure. In each film (except those with special indication), LiF nanoparticle, undoped ZnS/PVP QDs, and PVA matrix were used.

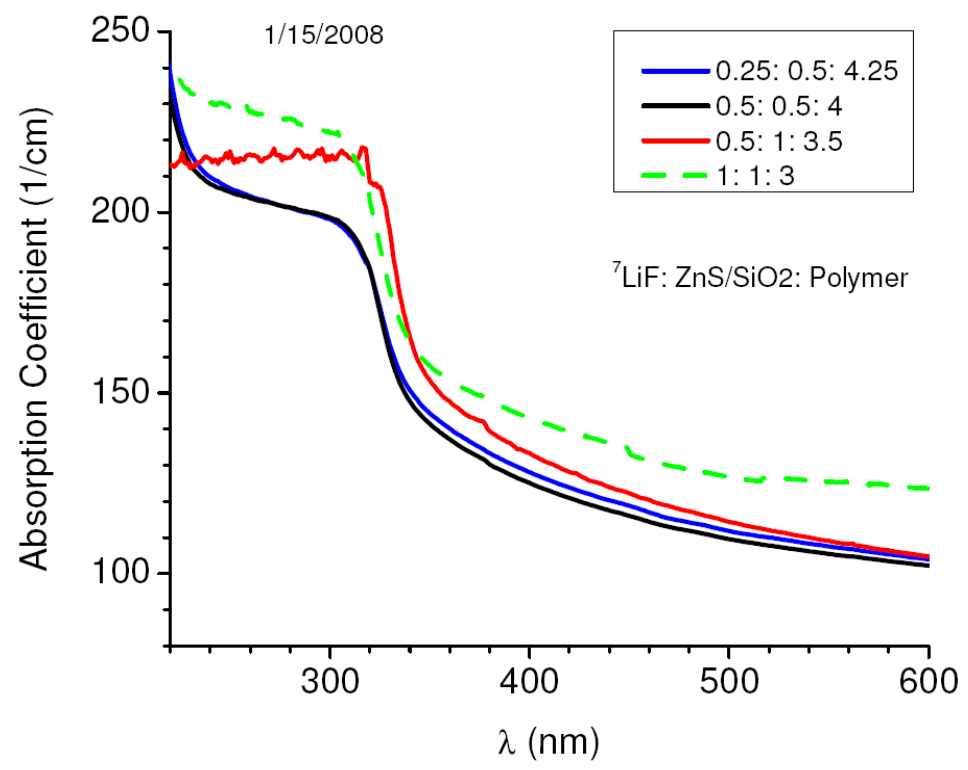

Figure 1-11. Optical absorption coefficient for several 7LiF: $\mathrm{ZnS} / \mathrm{SiO} 2 \mathrm{QD}$ : polymer nanocomposite films. The weight ratios are listed in the legend.

Figure 1-10 indicates that increasing nanoparticle concentrations results in the increase in the absorption coefficient at $420 \mathrm{~nm}$. While replacing LiF microparticles with nanoparticles, using $\mathrm{SiO}_{2}$ coated $\mathrm{ZnS}$ QDs can result in smaller optical absorption at $420 \mathrm{~nm}$. PartTec also tried to reduce the light absorption at $420 \mathrm{~nm}$ in $\mathrm{ZnS} / \mathrm{SiO}_{2}$ QD composite films by reducing the concentration of nanoparticles. The result is shown in Figure 1-11 
Final Report Next Generation Neutron Scintillators DOE Award: DE-FG02-07ER84884 based on Semiconductor Nanostructures

Photoluminescence quantum yields of the thick nanocomposite films $(0.1-0.3 \mathrm{~mm})$ in Figure 1-10 and Figure 1-11 are difficult to measure, since optical absorption induced by LiF nanoparticles (or microparticles) is significant in the total absorption coefficient. This effect causes the optical absorption by ZnS QDs less precise, and therefore, the calculated QY is not reliable. In addition, the large light scattering due to large concentrations of nanoparticles is also a concern, as it contributes to high backgrounds in the light absorption curves in thick films (Figure 1-10 and Figure 1-11). However, it is possible to reliably measure quantum yield when ZnS QDs are solely mixed with polymers and when thin films are fabricated $(<10 \mu \mathrm{m})$. In this case, the results are expected to be close to those measured in the dilute. Nevertheless, we believe that quantum yield of ZnS QDs measured in waterethanol solution is close to that in PVA matrix, since the wide band gap $\left(E_{\mathrm{g}}=5.4 \mathrm{eV}\right)$ of PVA matrix prohibits electrons or holes created by excitation to diffuse out of $\mathrm{ZnS}$ QDs (band gap $\left.E_{\mathrm{g}}=4.1 \mathrm{eV}\right)$.

\subsubsection{Neutron tests}

Several $\mathrm{ZnS}$ QDs $/{ }^{6} \mathrm{LiF}$ nanocomposite films were excited by thermal neutrons emitted from a plastic block in the front of a moderated Cf-252 source. The test was performed at the Neutron Facilities Development Division, Neutron Detector group Laboratory at ORNL. A photomultiplier tube (PMT) and a Multi-Channel Analyzer (MCA) board were used to collect pulse height spectra. An oscilloscope was used to collect light pulse shape spectra from the PMT. Figure 1-12 shows two examples of light pulses from a nanocomposite film with a ratio, by weight, of 0.5: 0.5: 4. Clearly, the pulse widths are in the range of 10-30 ns, close to that from UV excitation (Figure 1-2). It is shorter than neutron-induced light pulse decay times of Ce-doped ${ }^{6} \mathrm{Li}$-glass $(75 \mathrm{~ns})$ and $\mathrm{ZnS}: \mathrm{Ag} /{ }^{6} \mathrm{LiF}$ mciroparticle based scintillator (Table 1-1). This result is somewhat different from other conventional crystalline scintillators, whose light pulse decay times due to thermal neutrons are usually much longer than those excited from electrons (or gamma-rays, X-rays, UV sources) (van Eijk, 2004). More precise measurements will be conducted as part of this proposal. 
Final Report Next Generation Neutron Scintillators ～DOE Award: DE-FG02-07ER84884 based on Semiconductor Nanostructures

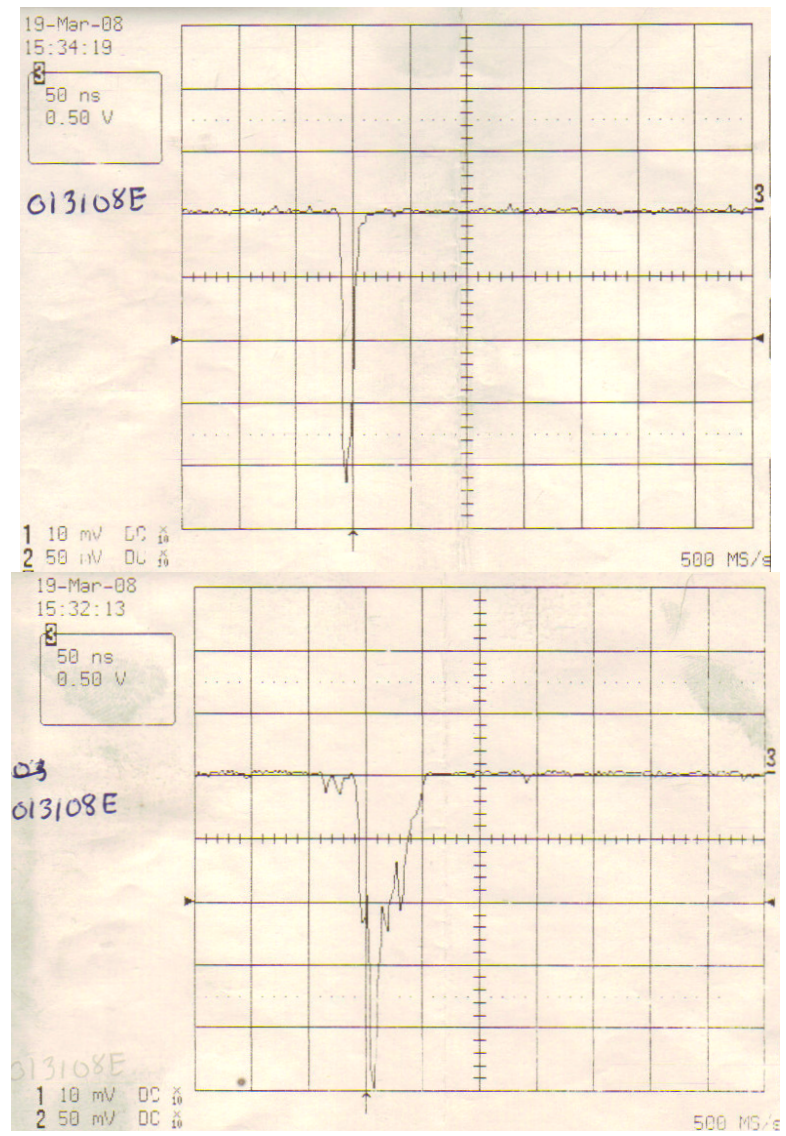

Figure 1-12. Two light pulse shape spectra induced by thermal/epithermal neutrons from an undoped $\mathrm{ZnS}$ QD/6LiF nanocompsite scintillator with thickness of $0.16 \mathrm{~mm}$. The scale of $\mathrm{x}$-axis is $50 \mathrm{~ns}$ per grid.

Figure 1-13 shows pulse height spectra of two ZnS QD composite scintillators under irradiation of thermal neutrons. In comparison with commercial BC704, PartTec's 013108E sample has lower light yield, since the fraction of ${ }^{6} \mathrm{LiF}+\mathrm{ZnS}$ QDs $(15-20 \%)$ is much lower than that of BC704 (80\%), and these ZnS QDs have a lower luminescence quantum yield $(\sim 10 \%)$ than Ag:ZnS microparticles. Two pulse height peaks can, however, be clearly seen in the spectra of the two nanocomposite samples, which will benefit future neutron-gamma discrimination. BC704 has no clear pulse peak as reported previously (Clergeau, 2002; Katagiri, 2004a), due to strong absorption and scattering to its own scintillation light. Figure 1-13 indicates that PartTec's nanocomposite scintillators, like GS20 ${ }^{6} \mathrm{Li}$-glass (Clergeau, 2002), have better energy resolution than microparticle $\mathrm{ZnS}: \mathrm{Ag} /{ }^{6} \mathrm{LiF}$ scintillator; though, PartTec agrees that the light yields of these nanocomposites need to be improved. This is one of main tasks PartTec will pursue during Phase II work. 
Final Report Next Generation Neutron Scintillators ～DOE Award: DE-FG02-07ER84884 based on Semiconductor Nanostructures

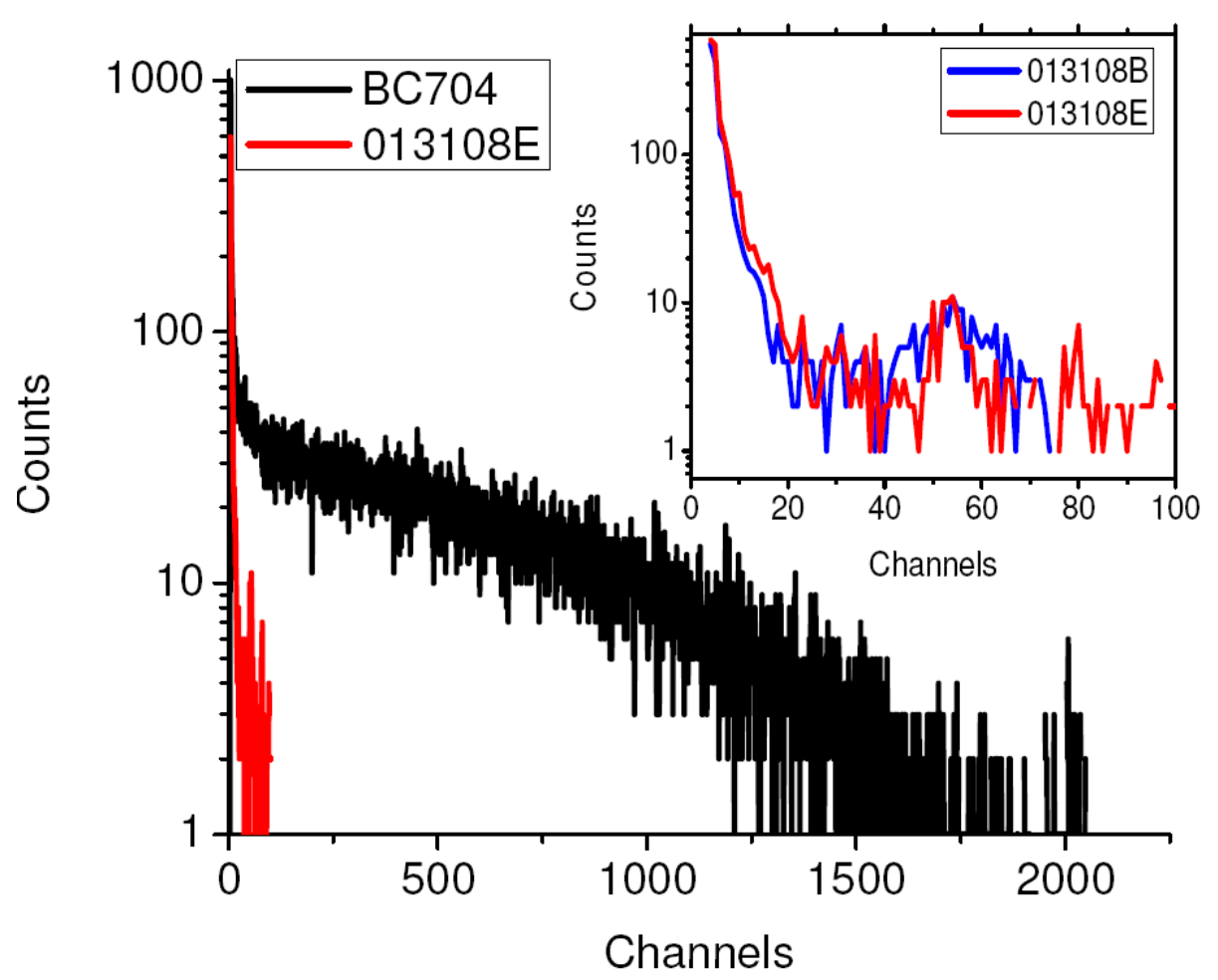

Figure 1-13. A pulse height spectrum of a $\mathrm{ZnS}$ QD nanocomposite scintillator under thermal neutron irradiation, in comparison with Saint-Gobain commercial product BC704. In the inserted figure, two pulse height spectra are shown for two nanocomposites, 010308B (weight ratio of LiF: ZnS QD: polymer =0.25: 0.5: 4.25), and 013108E (weight ratio=0.5: 0.5: 4).

\subsubsection{Conclusion}

The results reported here successfully demonstrate the technical feasibility of $\mathrm{ZnS}$ $\mathrm{QDs} /{ }^{6} \mathrm{LiF} /$ polymer composites as thermal neutron scintillators. PartTec has obtained stable ZnS QDs with a quantum yield of $17 \%$ induced by UV light, and light pulse decay lifetimes of 10-30 ns induced by both UV and neutrons. These lifetime values are much shorter than those of commercial $\mathrm{ZnS}$ microparticle and ${ }^{6} \mathrm{Li}$-glass scintillators. Clear pulse height peaks induced by neutron irradiation were seen for PartTec's ZnS nanocomposites. By adjusting the concentrations, particle size and degree of dispersion of $\mathrm{ZnS} \mathrm{QD} / 6 \mathrm{LiF}$ in a PVA matrix, the light absorption and light yield of films at 420-440 nm can be optimized. PartTec's novel scintillators will replace traditional ${ }^{6} \mathrm{Li}$-glass and $\mathrm{ZnS} /{ }^{6} \mathrm{LiF}: \mathrm{Ag}$ scintillators if the PL quantum yield can be improved above $30 \%$, and/or increase the transparency of present nanoscintillators.

Time and resources inhibited PartTec's total success in Phase I. For example, bulk doping preparations of $\mathrm{ZnS}$ QDs with $\mathrm{Ag}^{+}, \mathrm{Eu}^{3+}$ or $\mathrm{Ce}^{3+}$ QDs was impractical given those constraints, nor did they permit PartTec to measure systematically the change of PL decay lifetimes in different samples. PartTec will pursue these studies in the current proposal, as well as develop a better capping and dopant along with developing brighter and faster $\mathrm{ZnS}$ QD scintillators. 
Final Report Next Generation Neutron Scintillators based on Semiconductor Nanostructures

DOE Award: DE-FG02-07ER84884

\subsection{BIBLIOGRAPHY \& REFERENCES CITED}

Bol, A. A., R. van Beek, and A. Meijerink, On the incorporation of trivalent rare earth ions in II- VI semiconductor nanocrystals, Chem. Mater. 14, 1121 (2002).

Bol, A. A., and A. Meijerink, Luminescence quantum efficiency of nanocrystalline $\mathrm{ZnS}: \mathrm{Mn}^{2+}$ : surface passiviation and $\mathrm{Mn}^{2+}$ concentration, J. Phys. Chem. B 105, 10197 (2001).

Brus, L. E., Electron-electron and electron-hole interactions in small semiconductor crystallites: the size dependence of the lowest excited electronic state, J. Chem. Phys. 80. 4403 (1984).

Campbell, I. H., and B. K. Crone, Quantum-dot/organic semiconductor composites for radiation detection, Adv. Mater. 18, 77 (2006).

Cardona, M., and G. Harbeke, Optical properties and band structure of wurtzite-type crystals and rutile, Phys. Rev. 137, A1467 (1965).

Clergeau, J. F., et al., High position resolution neutron detector using a position sensitive photomultiplier tube. Proc. SPIE 4785, 254 (2002).

Cooper, R. G., I. Anderson, C. Britton, K. Crawford, M. L. Crow, P. DeLurgio, C. Hoffmann, D.Hutchinson, R. Klann, I. Naday, and G. Smith, "A program for neutron detector research and development", a SNS white paper (2003). See: http://www.sns.gov/pubs/index.shtml

Cooper, R.G., SNS detector plans, Nucl. Instrum Meth. A 529, 394 (2004).

Crow, M. L., J. P. Hodges, and R. G. Cooper, Shifting scintillator prototype large pixel wavelengthshifting fiber detector for the POWGEN3 powder diffractometer, Nucl. Instrum. Meth. A 529, 287 (2004).

Crowford, R. K., Position-sensitive detection of slow neutrons-survey of fundamental principles, Proc. SPIE, 1737, 210 (1992).

Cumberland, S. L., et al., Inorganic clusters as single source precursors for preparation of CdSe, $\mathrm{ZnSe}$, and CdSe/ZnS nanomaterials, Chem. Mater. 14, 1576 (2002).

Dean, P. J., Photoluminescence as a diagnostic of semiconductor, Prog. Cryst. Growth Character. 5, 89 (1982).

Demas, J. N., and G. A. Crosby, The measurement of photoluminescence quantum yields, a review, $J$. Phys. Chem. 75, 991 (1971).

Era, K., S. Shionoya, and Y. Washizawa, Mechanism of broad-band luminescences in ZnS phosphors, J. Phys. Chem. solids 29, 1827 and 1843(1968).

Feldmann, C., Polyol-mediated synthesis of nanoscale functional materials, Adv. Funct. Mater. 13, 101 (2003).

Gorin, A., et al., Development of scintillation imaging device for cold neutrons, Nucl. Instrum. Meth. A 479, 456 (2002).

Im, H.J., et al., Transparent matrix structures for detection of neutron particles based on di-ureasil xerogels, Appl. Phys. Lett. 84, 2448 (2004).

Jian, W., J. Zhuang, D. Zhang, J. Dai, W. Yang, and Y. Bai, Synthesis of highly luminescent and photostable ZnS:Ag nanocrystals under microwave radiation, Mater. Chem. Phys. 99, 494 (2006).

Johnson, J. A., et al., Eu-activated fluorochlorozirconate glass-ceramic scintillators, J. Appl. Phys. 100, 034701 (2006). 
Final Report Next Generation Neutron Scintillators ～DOE Award: DE-FG02-07ER84884 based on Semiconductor Nanostructures

Katagiri, M., et al., Scintillation materials for neutron imaging detectors, Nucl. Instrum. Meth. A 529, 274 (2004a).

Katagiri, M., et al., Neutron/ $\gamma$-ray discrimination characteristics of novel neutron scintillators, $\mathrm{Nucl}$. Instrum. Meth. A 529, 317 (2004b).

Kesanli, B., K. Long, K. Meyer, H. J. Im, and S. Dai, Highly efficient solid-state neutron scintillators based on hybrid sol-gel nanocomposite materials, Appl. Phys. Lett. 89, 214104 (2006).

Khosravi, et al., Green luminescence from copper dopped zinc sulphide quantum particles, Appl. Phys. Lett. 67, 2702 (1995).

Kubin, R. F., and A. N. Fletcher, Fluorescence quantum yields of some Rhodamine dyes, J. Lumin. 27, 455 (1981).

Letant, S. E. and T. F. Wang, Study of porous glass doped with quantum dots or laser dyes under alpha radiation, Appl. Phys. Lett. 88, 103110 (2006).

Lippens, P.E., and M. Lannoo, Calculation of the band gap for small CdS and $\mathrm{ZnS}$ crystallites, Phys. Rev. B 39, 10935 (1989).

Mansuy, C., J. M. Nedelec, and R. Mahiou, Molecular design of inorganic scintillators: from alkoxides to scintillation materials, J. Mater. Chem. 14, 3274 (2004).

Manzoor, K., S. R. Vadera, N. Kumar, and T. R. N. Kutty, Energy transfer from organic surface adsorbate-polyvinyl pyrrolidone molecules to luminescent centers in $\mathrm{ZnS}$ nanocrystals, Solid State Commun. 129, 469 (2004).

Swiatek, K. and M. Godlewski, Deactivation of photoionization excitation mechanism of rare-earth intraion emission in ZnS, Appl. Phys. Lett. 56, 2192 (1990).

Van Eijk, C. W. E., et al., Inorganic thermal- neutron scintillators, Nucl. Instrum. Meth. A 529, 260 (2004).

Wright, K., and R. A. Jackson, Computer simulation of the structure and defect properties of zinc sulfide, J. Mater. Chem. 5, 2037 (1995).

Yin, Y., and A. P. Alivisatos, Colloidal nanocrystal synthesis and the organic-inorganic interface, Nature 437, 664 (2005). 